\title{
Expressed and Private Opinion Dynamics on Influence Networks with Asynchronous Updating
}

\author{
Weiguo Xia ${ }^{1}$, Hong Liang ${ }^{1}$, and Mengbin $\mathrm{Ye}^{2}$
}

\begin{abstract}
In this paper, an asynchronous discrete-time opinion dynamics model on a social influence network is considered. At each time instant, a single individual activates and updates two state variables simultaneously. The individual's new private opinion is a weighted average of her current private opinion, the expressed opinions of her neighbors, and a constant prejudice. Meanwhile, the individual's new expressed opinion is equal to her current private opinion, altered due to a pressure to conform to the public opinion as perceived by the individual, being the average expressed opinion among her neighbors. We analyze the system for social networks which are rooted, and show that if no individual holds a prejudice, then a mild assumption on the activation sequence of the individuals guarantees convergence. In particular, the expressed and private opinions of all individuals converge to the same value exponentially fast, with two lower bounds on convergence speeds based on two different assumptions on the network topology. Simulations are provided to illustrate the result, and provide support to the conjecture that the system dynamics may converge even if individuals hold an existing prejudice.
\end{abstract}

\section{INTRODUCTION}

There has been a recent increase in interest among systems and control researchers on studying opinion dynamics models on social networks. Opinion dynamics is concerned with the study of how opinions form and evolve as individuals interact and exchange opinions on a social network [1].

A great majority of the literature on opinion dynamics grew from the seminal DeGroot model [2], which posits that each individual holds an opinion, being a real valued variable on a continuous interval, on some given topic. In a discrete-time process, individuals' opinions simultaneously evolve via a weighted averaging mechanism aimed at modeling a plausible method by which an individual processes exogenous learned information [3]. Since then, a number of different models, based on weighted averaging, have been proposed to expand the range of phenomena that can be predicted. Popular and notable contributions

This work was supported in part by the National Key R\&D Program of China under Grant 2018YFB1700102, the National Natural Science Foundation of China under Grants 61973051, 61911530696, the Fundamental Research Funds for the Central Universities under Grant DUT19ZD103, the Youth Star of Dalian Science and Technology (2018RQ51), the Australian Research Council DP160104500 and DP190100887, and Optus Business.

${ }^{1} \mathrm{~W}$. Xia and H. Liang are with the Key Laboratory of Intelligent Control and Optimization for Industrial Equipment of Ministry of Education and the School of Control Science and Engineering, Dalian University of Technology, Dalian, China. Emails: wgxiaseu@dlut.edu.cn, hongliang56@mail.dlut.edu.cn

${ }^{2} \mathrm{M}$. Ye is with the Optus-Curtin Centre of Excellence in Artificial Intelligence, Curtin University, Perth, Australia. Email: mengbin. yedcurtin.edu.au include the Friedkin-Johnsen model [4], the HegselmannKrause model [5], and the Altafini model [6], [7]. For further information, an extensive tutorial and summary of the key developments can be found in [8], [9].

Numerous studies in the literature of sociology, psychology and other related fields have identified that in many group situations, an individual may express an opinion that is not precisely her true private opinion, for a single given topic. Such a phenomenon may also be intuitive to the reader. In 1951, Solomon Asch illustrated in a set of seminal experiments how group peer pressure can distort an individual's judgment and expressed support on a provably true fact [10]. Many reasons exist for an individual to express an opinion different to her private opinion, including preference falsification [11], and impression management [12]. A common underlying theme is that individuals often feel $a$ pressure to conform to a perceived or imposed social norm within the group or social network they are interacting in.

Two recent works have been inspired by this body of literature, to develop models for expressed and private opinion dynamics. The work of Duggins [13] proposed a model in which individuals update asynchronously; the highly nonlinear complexities of the dynamics means detailed theoretical analysis is difficult, if not impossible. The work of Ye et. al. proposed a linear, synchronous Expressed and Private Opinion (EPO) model expanding from the Friedkin-Johnsen model and illustrated that the model could be used to capture the outcomes of Asch's experiments [14], and the phenomenon of pluralistic ignorance [15]. A few works have since expanded on the EPO model [16], [17].

One shortcoming of the EPO model, inherited from the Friedkin-Johsen and DeGroot models, is the synchronous nature of the dynamics. That is, all individuals simultaneously update their opinions. Asynchronous versions of many popular synchronous opinion dynamics models exist [18][22]. In such asynchronous models, it is typical that only a single individual is active at any time instant, and only her opinion(s) are changed, while all other individuals' opinions remain unchanged. The nature of the activation can vary; an individual may interact in a pairwise, gossip-like manner with a single neighbor, or may interact simultaneously with multiple others in her neighborhood. The activation sequence may occur over discrete-time instants in a deterministic or probabilistic manner, or individuals may have independent clocks driven by a Poisson process on a continuous time interval.

In this paper, we consider the EPO model under asynchronous updating, which more accurately reflects the way 
in which individuals interact in the real world. In particular, we assume that at any discrete time instant, a single individual activates and learns of all of her neighbors' expressed opinions. Then, the activated individual updates her private and expressed opinions simultaneously. The private opinion evolves as a weighted average of the individual's current private opinion, the expressed opinions of others, and a constant prejudice. The expressed opinion is the private opinion of the individual, altered by a pressure to conform to the average expressed opinion in her neighborhood. Over a network which is assumed to be rooted, two convergence results are obtained by assuming no individual is attached to her prejudice, including lower bounds on the convergence speeds. Simulations are provided to illustrate the theoretical results, and provide support to the conjecture that even if individuals are prejudiced, convergence can be established.

The rest of the paper is organized as follows. Section II provides mathematical notation, relevant aspects of graph theory, and introduces the opinion dynamics model. Section III provides theoretical results on convergence of the system. Simulations are presented in Section IV, and the paper is concluded in Section V.

\section{Preliminaries AND MOdel InTROduction}

To begin, we establish the mathematical notation to be used in this paper. The $n$-column vectors of all ones and zeros are given by $\mathbf{1}_{n}$ and $\mathbf{0}_{n}$, respectively. The $n \times n$ identity matrix is given by $I_{n}$. The $i^{\text {th }}$ canonical base unit vector of $\mathbb{R}^{n}$ is denoted as $\mathbf{e}_{i}$. We say that a matrix $A \in \mathbb{R}^{n \times n}$ is nonnegative (respectively positive) if all of its entries $a_{i j}$ are nonnegative (respectively positive), and is denoted by $A \geq 0$ and $A>0$ respectively. A square matrix $A \geq 0$ is said to be stochastic if, for all $i=1, \ldots, n$, there holds $\sum_{j=1}^{n} a_{i j}=$ 1. A stochastic matrix $A$ is a scrambling matrix if for any pair of distinct row indices $i$ and $j$, there always exists a column index $k$ such that both $p_{i k}$ and $p_{j k}$ are positive. For a stochastic matrix $A$, its coefficient of ergodicity $\tau(A)$ is defined as

$\tau(A)=\frac{1}{2} \max _{l, k} \sum_{s=1}^{n}\left|a_{l s}-a_{k s}\right|=1-\min _{l, k} \sum_{s=1}^{n} \min \left\{a_{l s}, a_{k s}\right\}$.

Note that $0 \leq \tau(A) \leq 1$, and $\tau(A)<1$ if and only if $A$ is a scrambling matrix [23]. Moreover, $\tau(A)=0$ if and only if $A$ is a rank-one matrix with all rows equal.

\section{A. Graph Theory}

For a square matrix $A \geq 0$, we can associate with it a directed graph $\mathcal{G}[A]=(\mathcal{V}, \mathcal{E}[A], A)$, where $\mathcal{V}=\left\{v_{1}, \ldots, v_{n}\right\}$ is the set of vertices of $\mathcal{G}[A]$. An edge $e_{i j}=\left(v_{i}, v_{j}\right)$ is in the set of ordered edges $\mathcal{E}[A] \subseteq \mathcal{V} \times \mathcal{V}$ if and only if $a_{j i}>0$. A self-loop for vertex $v_{i}$ exists if $e_{i i} \in \mathcal{E}[A]$. The edge $e_{i j}$ is said to be incoming with respect to $v_{j}$ and outgoing with respect to $v_{i}$. The incoming neighbor set and the outgoing neighbor set of $v_{i}$ are defined as $\mathcal{N}_{i}^{\text {in }}=\left\{v_{j} \in\right.$ $\left.\mathcal{V}:\left(v_{j}, v_{i}\right) \in \mathcal{E}[A]\right\}$ and $\mathcal{N}_{i}^{\text {out }}=\left\{v_{k} \in \mathcal{V}:\left(v_{i}, v_{k}\right) \in \mathcal{E}[A]\right\}$, respectively. A directed path is a sequence of edges of the form $\left(v_{p_{1}}, v_{p_{2}}\right),\left(v_{p_{2}}, v_{p_{3}}\right), \ldots$, where $v_{p_{i}} \in \mathcal{V}$ are distinct and $e_{p_{i} p_{i+1}} \in \mathcal{E}$. For a directed graph $\mathcal{G}[A]$, a vertex $v_{i}$ is a root of $\mathcal{G}[A]$ if for every other vertex $v_{j}$ of $\mathcal{G}[A]$, there exists a directed path from $v_{i}$ to $v_{j}$. We will say $\mathcal{G}[A]$ is rooted at $v_{i}$ if $v_{i}$ is a root, and $\mathcal{G}[A]$ is strongly rooted if every other vertex is an outgoing neighbor of $v_{i}$.

Let $\mathcal{G}_{1}$ and $\mathcal{G}_{2}$ be two directed graphs with the same vertex set $\left\{v_{1}, \ldots, v_{n}\right\}$. By the composition of $\mathcal{G}_{1}$ with $\mathcal{G}_{2}$, written $\mathcal{G}_{2} \circ \mathcal{G}_{1}$, we mean the directed graph with the vertex set $\left\{v_{1}, \ldots, v_{n}\right\}$ and the edge set defined such that $\left(v_{i}, v_{j}\right)$ is an edge of the composition if and only if there exists a vertex $v_{k}$ such that $\left(v_{i}, v_{k}\right)$ is an edge of $\mathcal{G}_{1}$ and $\left(v_{k}, v_{j}\right)$ is an edge of $\mathcal{G}_{2}$.

\section{B. Expressed and Private Opinion Model}

The Expressed and Private Opinion (EPO) model in the asynchronous updating framework will now be presented formally, followed by discussions on its motivation; further details are presented in [15]. Consider a population of $n \geq 2$ individuals, indexed by $i \in \mathcal{I} \triangleq\{1, \ldots, n\}$, interacting in a social network at discrete-time instants $t=0,1, \ldots$ We assume that each individual $i \in \mathcal{I}$ has a private opinion, $x_{i}(t) \in \mathbb{R}$, and an expressed opinion, $\hat{x}_{i}(t) \in \mathbb{R}$, on a given topic that may evolve over time $t$. In general, an individual may choose to express an opinion $\hat{x}_{i}(t)$ not equal to her private opinion $x_{i}(t)$, for a number of different reasons as recorded by numerous studies in the social sciences. For example, Timur Kuran has identified that preference falsification can occur when an individual falsifies her opinion, to garner favours or subconsciously due to social pressure to follow a norm [11].

Individuals discuss and exchange expressed opinions over a network, modelled by a graph $\mathcal{G}[W]$, according to the following asynchronous dynamics. At each time $t$, a single individual $i$ is activated and updates her private and expressed opinions simultaneously according to

$$
\begin{aligned}
& x_{i}(t+1)=\lambda_{i} w_{i i} x_{i}(t)+\lambda_{i} \sum_{j=1}^{n} w_{i j} \hat{x}_{j}(t)+\left(1-\lambda_{i}\right) u_{i} \\
& \hat{x}_{i}(t+1)=\phi_{i} x_{i}(t)+\left(1-\phi_{i}\right) z_{i}(t) .
\end{aligned}
$$

The opinions of all other individuals are unchanged: for individual $j \neq i, x_{j}(t+1)=x_{j}(t)$ and $\hat{x}_{j}(t+1)=\hat{x}_{j}(t)$. In the sequel, we place clear assumptions on the frequency of activation for the individuals, but we first detail the parameters in Eq. (2). The influence weight $w_{i j} \geq 0$ captures the influence of individual $j$ 's expressed opinion $\hat{x}_{j}(t)$ in Eq. (2a), and satisfies $\sum_{j=1}^{n} w_{i j}=1$ for all $i \in \mathcal{I}$. The constant $u_{i} \in \mathbb{R}$ is termed the prejudice of individual $i$, and her susceptibility to influence is captured by the constant $\lambda_{i} \in[0,1]$. Thus $1-\lambda_{i}$ measures individual $i$ 's attachment to the prejudice $u_{i}$, with the most common choice being $u_{i}=x_{i}(0)$. The term $z_{i}(t)$ is called the public opinion as perceived by individual $i$, and is defined as

$$
z_{i}(t) \triangleq \sum_{j=1}^{n} m_{i j} \hat{x}_{j}(t)
$$


where the weights $m_{i j} \geq 0$ satisfy $\sum_{j=1}^{n} m_{i j}=1$.

The term $z_{i}(t)$ represents the group norm or standard opinion of the social network as seen by individual $i$, so that the constant $\phi_{i} \in[0,1]$ is the resilience to the pressures to conform to $z_{i}(t)$. In general, $m_{i j}$ and $w_{i j}$ are not the same, and it may even be the case that one is zero and the other positive. This is to reflect the fact that an individual $i$ 's private opinion may be shaped by one set of individuals in the social network, but may feel a pressure to conform to a different set of individuals (or to apply different weights).

We discussed above that many reasons exist for an individual to express an opinion different to her private opinion. The evolution in Eq. (2b) is just one possible method for individual $i$ to determine $\hat{x}_{i}(t+1)$, but it is one which has found significant support from the social science literature. Specifically, Eq. (2b) captures individual $i$ altering her private opinion to be closer to local public opinion $z_{i}(t)$, with $\left(1-\phi_{i}\right) z_{i}(t)$ representing the social "force" exerted. Such a pressure to conform towards the perceived group standard has been theorised, and supported by empirical evidence [10], [24], [25]. It has been found that such pressures can arise from unspoken traditions [26], a fear of appearing different [10], or a desire to maintain a positive impression as seen by others (impression management) [12].

To conclude, we formalise a standing assumption that will be enforced for the rest of this paper.

Assumption 1. There exists a finite positive integer $T$ such that, for any $t \geq 0$, every individual activates at least once in the interval $t, t+1, \ldots, t+T-1$.

We remark that the assumption on the activation of individual agents is standard in multi-agent systems, and agent-based models. One further assumption on the system parameters is presented, for use in the subsequent analysis.

Assumption 2. For stochastic matrices $W$ and $M, w_{i j}>0$ if and only if $m_{i j}>0$. Furthermore, there holds $\lambda_{i}=1, \phi_{i} \in$ $(0,1), \forall i \in \mathcal{I}$.

We need the following lemmas to derive our main results.

Lemma 1. [23] For stochastic matrices $A_{1}$ and $A_{2}$, the following inequality holds for the coefficient of ergodicity

$$
\tau\left(A_{1} A_{2}\right) \leq \tau\left(A_{1}\right) \tau\left(A_{2}\right)
$$

Lemma 2. Let $\mathcal{G}_{1}, \mathcal{G}_{2}$, and $\mathcal{G}_{3}$ be graphs with the same vertex set $\left\{v_{1}, \ldots, v_{n}\right\}$ and each vertex has a self-loop in $\mathcal{G}_{i}$ for $i=1,2,3$. If $\left(v_{i}, v_{j}\right)$ is an edge in $\mathcal{G}_{2}$, then it is also an edge in $\mathcal{G}_{3} \circ \mathcal{G}_{2} \circ \mathcal{G}_{1}$.

Proof. For each $i \in \mathcal{I},\left(v_{i}, v_{i}\right)$ is an edge in $\mathcal{G}_{1}$. Since $\left(v_{i}, v_{j}\right)$ is an edge in $\mathcal{G}_{2}$, it is also an edge in $\mathcal{G}_{2} \circ \mathcal{G}_{1}$. The conclusion follows from the fact that $\left(v_{j}, v_{j}\right)$ is an edge in $\mathcal{G}_{3}$.

\section{MAIN RESULTS}

In this section, we analyse the evolution of the opinion dynamics. To begin, we write the dynamics of the entire social network. It is convenient to first introduce definitions of certain matrices which will be useful toward this end. Let
$S_{i}=I_{n}-\mathbf{e}_{i} \mathbf{e}_{i}^{\top}$ be the identity matrix with the $i$ th diagonal entry set equal to zero. Let $\tilde{W}$ be a diagonal matrix with $i$ th diagonal entry equal to $w_{i i}$, and $\hat{W} \geq 0$ be a matrix with 0 on its diagonals, and $\hat{w}_{i j}=w_{i j}$ for all $j \neq i$. Thus, $W=\tilde{W}+\hat{W}$. Further, define $\Lambda=\operatorname{diag}\left(\lambda_{1}, \ldots, \lambda_{n}\right)$ and $\Phi=\operatorname{diag}\left(\phi_{1}, \ldots, \phi_{n}\right)$.

Let $x=\left[x_{1}, \ldots, x_{n}\right]^{\top} \in \mathbb{R}^{n}$ and $\hat{x}=\left[\hat{x}_{1}, \ldots, \hat{x}_{n}\right]^{\top} \in \mathbb{R}^{n}$ and $u=\left[u_{1}, \ldots, u_{n}\right]^{\top} \in \mathbb{R}^{n}$ denote the vector of all private opinions and all expressed opinions and all prejudices, respectively. Then, define $\zeta=\left[x^{\top}, \hat{x}^{\top}\right]^{\top}$ as the vector of all opinions among the individuals in the social network. Next, define $\sigma(t): \mathbb{Z}_{\geq 0} \rightarrow \mathcal{I}$ as the activation sequence of individuals in the social network, so that individual $i$ is activated at time $t$ if and only if $\sigma(t)=i$. The dynamics in Eq. (2) for all $i=1, \ldots, n$ can be equivalently expressed as

$$
\zeta(t+1)=P_{\sigma(t)} \zeta(t)+U_{\sigma(t)}
$$

where, for all $i \in \mathcal{I}$, we define

$$
P_{i}=\left[\begin{array}{cc}
S_{i}+\mathbf{e}_{i} \mathbf{e}_{i}^{\top} \Lambda \tilde{W} & \mathbf{e}_{i} \mathbf{e}_{i}^{\top} \Lambda \hat{W} \\
\mathbf{e}_{i} \mathbf{e}_{i}^{\top} \Phi & S_{i}+\mathbf{e}_{i} \mathbf{e}_{i}^{\top}\left(I_{n}-\Phi\right) M
\end{array}\right] \in \mathbb{R}^{2 n \times 2 n},
$$

and

$$
U_{i}=\left[\begin{array}{c}
\mathbf{e}_{i} \mathbf{e}_{i}^{\top}\left(I_{n}-\Lambda\right) u \\
\mathbf{0}_{n}
\end{array}\right] \in \mathbb{R}^{2 n} .
$$

For the case when $\lambda_{i}=1$ for all $i \in \mathcal{I}$, as in Assumption 2, $P_{i}$ becomes

$$
P_{i}=\left[\begin{array}{cc}
S_{i}+\mathbf{e}_{i} \mathbf{e}_{i}^{\top} \tilde{W} & \mathbf{e}_{i} \mathbf{e}_{i}^{\top} \hat{W} \\
\mathbf{e}_{i} \mathbf{e}_{i}^{\top} \Phi & S_{i}+\mathbf{e}_{i} \mathbf{e}_{i}^{\top}\left(I_{n}-\Phi\right) M
\end{array}\right],
$$

and $U_{i}=\mathbf{0}_{2 n}$ is a vector of zeros. Let the vertex set of $\mathcal{G}[W]$ be denoted by $\left\{s_{1}, \ldots, s_{n}\right\}$. For the graph $\mathcal{G}\left[P_{i}\right]$, let the vertex set be denoted by $\left\{v_{1}, \ldots, v_{n}, v_{n+1}, \ldots, v_{2 n}\right\}$ corresponding to the state $\zeta_{1}, \zeta_{2}, \ldots, \zeta_{2 n}$, respectively. If $w_{i j}>0$ if and only if $m_{i j}>0$, and $\phi_{i} \in(0,1), \forall i \in \mathcal{I}$, then we have the following observations.

(O1) The vertices $\left\{v_{j}, v_{n+j}, j \in \mathcal{I} \backslash\{i\}\right\}$ have self-loops in the graph $\mathcal{G}\left[P_{i}\right]$ for all $i \in \mathcal{I}$. The vertices $v_{i}$ and $v_{n+i}$ have self-loops in $\mathcal{G}\left[P_{i}\right]$ if and only if $w_{i i}>0$.

(O2) The edge $\left(v_{i}, v_{n+i}\right) \in \mathcal{E}\left[P_{i}\right]$ for all $i \in \mathcal{I}$.

(O3) If $\left(s_{i}, s_{j}\right) \in \mathcal{E}[W]$ for some $j \in \mathcal{I} \backslash\{i\}$, then $\left(v_{n+i}, v_{j}\right)$ and $\left(v_{n+i}, v_{n+j}\right) \in \mathcal{E}\left[P_{j}\right]$, as one can infer from the upper block part $\mathbf{e}_{j} \mathbf{e}_{j}^{\top} \hat{W}$ of $P_{j}$.

Theorem 1. Suppose that Assumption 1 and Assumption 2 hold. If for all $i \in \mathcal{I}, w_{i i}>0$ and the network $\mathcal{G}[W]$ is rooted, then for the system (2), the expressed opinions and the private opinions of all the individuals reach the same value exponentially fast at a rate no slower than

$$
\left(1-\alpha^{n T}\right)^{\frac{1}{n T}}
$$

where $\alpha$ is the smallest value of the nonzero entries among all the matrices $P_{i}, i \in \mathcal{I}$.

Proof. The matrix $P_{i}$ is given by (8) and $U_{i}$ is a zero vector under Assumption 2 and one can verify that $P_{i}$ is a stochastic matrix since $W$ and $M$ are both stochastic. Noting that $w_{i i}, m_{i i}>0$ and $\phi_{i} \in(0,1)$ for all $i \in \mathcal{I}$, it follows from 
Observation $\mathrm{O} 1$ that the vertices in the graph $\mathcal{G}\left[P_{i}\right]$ all have self-loops.

The solution to Eq. (5) is given by

$$
\zeta(t)=\Pi_{k=0}^{t} P_{\sigma(k)} \zeta(0) .
$$

The matrix product $\Pi_{k=0}^{t} P_{\sigma(k)}$ is a stochastic matrix for all $t \geq 0$. The main idea of the theorem proof is to show that $\Pi_{k=0}^{t} P_{\sigma(k)}$ converges to a rank-one stochastic matrix $\mathbf{1}_{2 n} v^{\top}$ for some nonnegative vector $v \in \mathbb{R}_{>0}^{2 n}$, exponentially fast as $t \rightarrow \infty$. This in conjunction with the above equation implies that the state $\zeta(t)$ converges to $c \mathbf{1}_{2 n}$ exponentially fast for $c=v^{\top} \zeta(0) \in \mathbb{R}$. To do so, we will prove that for all $t \geq 0$, $\mathcal{G}\left[P_{\sigma(t+(n-1) T-1)} \cdots P_{\sigma(t)}\right]$ is strongly rooted at some vertex and hence the matrix $P_{\sigma(t+(n-1) T-1)} \cdots P_{\sigma(t)}$ has a positive column and is naturally a scrambling matrix.

Consider an arbitrary time instant $t \geq 0$. Suppose that $s_{i}$ is a root of the graph $\mathcal{G}[W]$. By Lemma 3 in [27], $\mathcal{G}\left[P_{\sigma(t+(n-1) T-1)} \cdots P_{\sigma(t)}\right]$ has the same graph structure as $\mathcal{G}\left[P_{\sigma(t+(n-1) T-1)}\right] \circ \cdots \circ \mathcal{G}\left[P_{\sigma(t)}\right]$. We will prove that $\left(v_{i}, v_{j}\right)$ and $\left(v_{i}, v_{n+j}\right)$ are edges in $\mathcal{G}\left[P_{\sigma(t+(n-1) T-1)}\right] \circ$ $\cdots \circ \mathcal{G}\left[P_{\sigma(t)}\right]$ for all $j \in \mathcal{I}$ and $t \geq 0$, implying that $\mathcal{G}\left[P_{\sigma(t+(n-1) T-1)} \cdots P_{\sigma(t)}\right]$ is strongly rooted at $v_{i}$. In the following, we consider two cases.

Case 1: The index $j=i$. Since the vertices in $\mathcal{G}\left[P_{\sigma(t)}\right]$ all have self-loops for all $t \geq 0, v_{i}$ has a self-loop in $\mathcal{G}\left[P_{\sigma(t+(n-1) T-1)}\right] \circ \cdots \circ \mathcal{G}\left[P_{\sigma(t)}\right]$. From Assumption 1, there exists some $t_{i}^{*}$ satisfying $t \leq t_{i}^{*} \leq t+T-1$, such that $\sigma\left(t_{i}^{*}\right)=i$, i.e. individual $i$ is activated at time $t_{i}^{*}$. It follows from Observation $\mathrm{O} 2$ that $\left(v_{i}, v_{n+i}\right) \in \mathcal{E}\left[P_{\sigma\left(t_{i}^{*}\right)}\right]$ and then $\left(v_{i}, v_{n+i}\right)$ is an edge in the graph $\mathcal{G}\left[P_{\sigma(t+T-1)}\right] \circ \cdots \circ \mathcal{G}\left[P_{\sigma(t)}\right]$ and also in $\mathcal{G}\left[P_{\sigma(t+n T-1)} \cdots P_{\sigma(t)}\right]$ by Lemma 2 .

Case 2: The index $j \neq i$. Suppose that $\left(s_{i_{0}}, s_{i_{1}}\right), \ldots,\left(s_{i_{r-1}}, s_{i_{r}}\right)$ is one shortest path with path length $r \geq 1$, from $s_{i}$ to $s_{j}$ in the graph $\mathcal{G}[W]$, where $i_{0}=i$ and $i_{r}=j$. Since $\mathcal{G}[W]$ is rooted, the shortest path from the root $s_{i}$ to any other vertex $s_{j}, j \in \mathcal{I} \backslash\{i\}$, is of path length at most $n-1$ and therefore $r \leq n-1$. Since Assumption 1 holds, there exists some $t_{1}^{*}$ satisfying $t+T \leq t_{1}^{*} \leq t+2 T-1$, such that $\sigma\left(t_{1}^{*}\right)=i_{1}$, i.e. individual $i_{1}$ is activated at time $t_{1}^{*}$. Since $\left(s_{i_{0}}, s_{i_{1}}\right) \in \mathcal{E}[W]$, it follows from Observation O3 that $\left(v_{n+i_{0}}, v_{i_{1}}\right)$ and $\left(v_{n+i_{0}}, v_{n+i_{1}}\right) \in \mathcal{E}\left[P_{\sigma\left(t_{1}^{*}\right)}\right]$ and so the two edges belong to the edge set of the graph composition $\mathcal{G}\left[P_{\sigma(t+2 T-1)}\right] \circ \cdots \circ \mathcal{G}\left[P_{\sigma(t+T)}\right]$ as well, in view of Lemma 2. In case 1 , it was shown that $\left(v_{i_{0}}, v_{n+i_{0}}\right)$ is an edge in the graph $\mathcal{G}\left[P_{\sigma(t+T-1)}\right] \circ \cdots \circ \mathcal{G}\left[P_{\sigma(t)}\right]$. Taking the composition of $\mathcal{G}\left[P_{\sigma(t+T-1)}\right] \circ \cdots \circ \mathcal{G}\left[P_{\sigma(t)}\right]$ with $\mathcal{G}\left[P_{\sigma(t+2 T-1)}\right] \circ \cdots \circ \mathcal{G}\left[P_{\sigma(t+T)}\right]$, we conclude that $\left(v_{i_{0}}, v_{i_{1}}\right)$ and $\left(v_{i_{0}}, v_{n+i_{1}}\right)$ are edges in $\mathcal{G}\left[P_{\sigma(t+2 T-1)}\right] \circ \cdots \circ \mathcal{G}\left[P_{\sigma(t)}\right]$. It follows from Lemma 2 that they are also edges in $\mathcal{G}\left[P_{\sigma(t+n T-1)}\right] \circ \cdots \circ \mathcal{G}\left[P_{\sigma(t)}\right]$. By induction, one can derive that $\left(v_{i}, v_{j}\right)$ and $\left(v_{i}, v_{n+j}\right)$ are edges in $\mathcal{G}\left[P_{\sigma(t+(r+1) T-1)}\right] \circ \cdots \circ \mathcal{G}\left[P_{\sigma(t)}\right]$. Since $r \leq n-1,\left(v_{i}, v_{j}\right)$ and $\left(v_{i}, v_{n+j}\right)$ are edges in $\mathcal{G}\left[P_{\sigma(t+n T-1)}\right] \circ \cdots \circ \mathcal{G}\left[P_{\sigma(t)}\right]$ from Lemma 2.

To conclude, we have established that $\mathcal{G}\left[P_{\sigma(t+n T-1)}\right] \circ$ $\cdots \circ \mathcal{G}\left[P_{\sigma(t)}\right]$ is strongly rooted at $v_{i}$, and so is $\mathcal{G}\left[P_{\sigma(t+n T-1)} \cdots P_{\sigma(t)}\right]$. Then, the matrix product
$P_{\sigma(t+n T-1)} \cdots P_{\sigma(t)}$ is a scrambling matrix. By the definition of coefficient of ergodicity, it follows that

$$
\tau\left(\Pi_{k=t}^{t+n T-1} P_{\sigma(k)}\right) \leq 1-\alpha^{n T} .
$$

By Lemma 1 and the fact that the coefficient of ergodicity is no greater than 1 , we obtain that for any $t \geq 0$,

$$
\begin{aligned}
\tau\left(\Pi_{k=0}^{t} P_{\sigma(k)}\right) & \leq \tau\left(\Pi_{k=0}^{\left\lfloor\frac{t}{n T}\right\rfloor n T-1} P_{\sigma(k)}\right) \\
& \leq\left(1-\alpha^{n T}\right)^{\left\lfloor\frac{t}{n T}\right\rfloor} \\
& \leq\left(1-\alpha^{n T}\right)^{\frac{t}{n T}-1},
\end{aligned}
$$

where $\left\lfloor\frac{t}{n T}\right\rfloor$ represents the largest integer less than or equal to $\frac{t}{n T}$ and $\Pi_{k=0}^{-1} P_{\sigma(k)}$ is defined as $P_{\sigma(0)}$. This implies that

$$
\lim _{t \rightarrow \infty} \tau\left(\Pi_{k=0}^{t} P_{\sigma(k)}\right) \leq \lim _{t \rightarrow \infty}\left(1-\alpha^{n T}\right)^{\frac{t}{n T}-1}=0 .
$$

According to the property of the coefficient of ergodicity stated below Eq. (1), the matrix product $\Pi_{k=0}^{t} P_{\sigma(k)}$ converges to a rank-one matrix exponentially fast as $t \rightarrow \infty$ and thus the conclusion follows.

Theorem 2. Suppose that Assumption 1 and Assumption 2 hold. If for some $i \in \mathcal{I}, w_{i i}>0$ and the network $\mathcal{G}[W]$ is rooted at $s_{i}$, then for the system (2), the expressed opinions and the private opinions of all the individuals reach the same value exponentially fast at a rate no slower than

$$
\left(1-\alpha^{2 n T}\right)^{\frac{1}{2 n T}},
$$

where $\alpha$ is the smallest value of the nonzero entries among all the matrices $P_{i}, i \in \mathcal{I}$.

Proof. Note that $P_{i}$ is given by (8) and $U_{i}$ is a zero vector under Assumption 2, but the diagonal entries of $P_{i}$ are not necessarily all positive since $w_{i i}$ and $m_{i i}$ can be zero. The main idea to prove the theorem is similar to that in Theorem 1, which is to show that $\prod_{k=0}^{t} P_{\sigma(k)}$ converges to a rank-one stochastic matrix exponentially fast as $t \rightarrow \infty$. To do so, we prove that for all $t \geq 0, \mathcal{G}\left[P_{\sigma(t+2 n T-1)} \cdots P_{\sigma(t)}\right]$ is strongly rooted at some vertex and hence the matrix $P_{\sigma(t+(n-1) T-1)} \cdots P_{\sigma(t)}$ has a positive column and is naturally a scrambling matrix.

Consider an arbitrary time instant $t \geq 0$. Suppose that $w_{i_{0} i_{0}}>0$ and the vertex $s_{i_{0}}, i_{0} \in \mathcal{I}$ is a root in $\mathcal{G}[W]$. Let $t_{0}, t \leq t_{0} \leq t+T-1$, be the first time instant when the agent $i_{0}$ activates after $t$. Then it follows from Observations $\mathrm{O} 1$ and $\mathrm{O} 2$ that $\left(v_{i_{0}}, v_{i_{0}}\right)$ and $\left(v_{n+i_{0}}, v_{n+i_{0}}\right)$ and $\left(v_{i_{0}}, v_{n+i_{0}}\right)$ are in $\mathcal{E}\left[P_{\sigma\left(t_{0}\right)}\right]$. Since $w_{i_{0} i_{0}}>0$, it follows from Observation $\mathrm{O} 1$ that for all $j \in \mathcal{I},\left(v_{i_{0}}, v_{i_{0}}\right)$ and $\left(v_{n+i_{0}}, v_{n+i_{0}}\right) \in \mathcal{E}\left[P_{j}\right]$; in contrast, if $w_{k k}=0$ for some $k \in \mathcal{I}$, then $\left(v_{k}, v_{k}\right)$ and $\left(v_{n+k}, v_{n+k}\right)$ are not in $\mathcal{E}\left[P_{k}\right]$. From the definition of graph composition, it follows from above that $\left(v_{i_{0}}, v_{i_{0}}\right)$ and $\left(v_{n+i_{0}}, v_{n+i_{0}}\right)$ and $\left(v_{i_{0}}, v_{n+i_{0}}\right)$ are edges in $\mathcal{G}\left[P_{j}\right] \circ \mathcal{G}\left[P_{\sigma\left(t_{0}\right)}\right]$. By induction, it follows that $\left(v_{i_{0}}, v_{i_{0}}\right)$ and $\left(v_{n+i_{0}}, v_{n+i_{0}}\right)$ and $\left(v_{i_{0}}, v_{n+i_{0}}\right)$ are in the composition $\mathcal{G}\left[P_{\sigma(t)}\right] \circ G\left[P_{\sigma(t-1)}\right] \circ \cdots \circ \mathcal{G}\left[P_{\sigma\left(t_{0}\right)}\right]$ for all $t \geq t_{0}$.

Suppose that $s_{i_{j}}$ are all outgoing neighbors of $s_{i_{0}}$ on $\mathcal{G}[W]$, for $j=1, \ldots, r$. Let $t_{1}$ be the first time instant after $t_{0}$ when the outgoing neighbor $s_{i_{1}}$ of $s_{i_{0}}$ activates. 
Then $\left(v_{n+i_{0}}, v_{i_{1}}\right)$ and $\left(v_{n+i_{0}}, v_{n+i_{1}}\right) \in \mathcal{E}\left[P_{\sigma\left(t_{1}\right)}\right]$ according to Observation O3. The presence of a self-loop at $v_{n+i_{0}}$ in $\mathcal{G}\left[P_{\sigma\left(t_{1}-1\right)}\right] \circ \ldots \circ \mathcal{G}\left[P_{\sigma\left(t_{0}\right)}\right]$ ensures that $\left(v_{n+i_{0}}, v_{i_{1}}\right)$ and $\left(v_{n+i_{0}}, v_{n+i_{1}}\right)$ belong to the edge set of $\mathcal{G}\left[P_{\sigma\left(t_{1}\right)}\right] \circ$ $\mathcal{G}\left[P_{\sigma\left(t_{1}-1\right)}\right] \circ \cdots \circ \mathcal{G}\left[P_{\sigma\left(t_{0}\right)}\right]$. For any $j \in \mathcal{I} \backslash\left\{i_{1}\right\}$, one has that $\left(v_{n+i_{0}}, v_{i_{1}}\right)$ and $\left(v_{n+i_{0}}, v_{n+i_{1}}\right)$ are edges in $\mathcal{G}\left[P_{j}\right] \circ$ $\mathcal{G}\left[P_{\sigma\left(t_{1}\right)}\right] \circ \cdots \circ \mathcal{G}\left[P_{\sigma\left(t_{0}\right)}\right]$ since $\left(v_{i_{1}}, v_{i_{1}}\right)$ and $\left(v_{n+i_{1}}, v_{n+i_{1}}\right) \in$ $\mathcal{E}\left[P_{j}\right]$. Noting that $\left(v_{n+i_{0}}, v_{i_{1}}\right)$ and $\left(v_{n+i_{0}}, v_{n+i_{1}}\right) \in \mathcal{E}\left[P_{i_{1}}\right]$ and $\left(v_{n+i_{0}}, v_{n+i_{0}}\right)$ is an edge in $\mathcal{G}\left[P_{\sigma\left(t_{1}\right)}\right] \circ \cdots \circ \mathcal{G}\left[P_{\sigma\left(t_{0}\right)}\right]$, it holds that $\left(v_{n+i_{0}}, v_{i_{1}}\right)$ and $\left(v_{n+i_{0}}, v_{n+i_{1}}\right)$ are edges in $\mathcal{G}\left[P_{j}\right] \circ \mathcal{G}\left[P_{\sigma\left(t_{1}\right)}\right] \circ \cdots \circ \mathcal{G}\left[P_{\sigma\left(t_{0}\right)}\right]$ as well when $j=i_{1}$. By induction, the two edges are in the edge set of the graph composition $\mathcal{G}\left[P_{\sigma(t)}\right] \circ \mathcal{G}\left[P_{\sigma(t-1)}\right] \circ \cdots \circ \mathcal{G}\left[P_{\sigma\left(t_{0}\right)}\right]$ for all $t \geq t_{1}$.

We remark that the above argument applies to every outgoing neighbors of $i_{0}$. Using the fact that every individual activates at least once in every $T$ steps, it follows that the graph composition $\mathcal{G}\left[P_{\sigma\left(t_{0}+T-1\right)}\right] \circ \cdots \circ \mathcal{G}\left[P_{\sigma\left(t_{0}\right)}\right]$ contains the edges $\left(v_{n+i_{0}}, v_{j}\right)$ and $\left(v_{n+i_{0}}, v_{n+j}\right)$ for all $j=i_{1}, i_{2}, \ldots, i_{r}$.

Let $k_{1}, \ldots, k_{p} \in \mathcal{I} \backslash\left\{i_{0}, \ldots, i_{r}\right\}$ be the outgoing neighbors of $s_{i_{1}}$. Let $t_{2}$ be the first time instant after $t_{0}+T-$ 1 when the outgoing neighbor $k_{1}$ of $s_{i_{1}}$ activates. Then $\left(v_{n+i_{1}}, v_{k_{1}}\right)$ and $\left(v_{n+i_{1}}, v_{n+k_{1}}\right) \in \mathcal{E}\left[P_{\sigma\left(t_{2}\right)}\right]$. Due to the presence of $\left(v_{n+i_{0}}, v_{n+i_{1}}\right)$ in $\mathcal{G}\left[P_{\sigma\left(t_{2}-1\right)}\right] \circ \cdots \circ \mathcal{G}\left[P_{\sigma\left(t_{0}\right)}\right]$, it follows that $\left(v_{n+i_{0}}, v_{k_{1}}\right)$ and $\left(v_{n+i_{0}}, v_{n+k_{1}}\right)$ are in the edge set of $\mathcal{G}\left[P_{\sigma\left(t_{2}\right)}\right] \circ \mathcal{G}\left[P_{\sigma\left(t_{2}-1\right)}\right] \circ \cdots \circ \mathcal{G}\left[P_{\sigma\left(t_{0}\right)}\right]$. For any $j \in \mathcal{I} \backslash\left\{k_{1}\right\}$, one can similarly show that $\left(v_{n+i_{0}}, v_{k_{1}}\right)$ and $\left(v_{n+i_{0}}, v_{n+k_{1}}\right)$ are edges in $\mathcal{G}\left[P_{j}\right] \circ \mathcal{G}\left[P_{\sigma\left(t_{2}\right)}\right] \circ \cdots \circ$ $\mathcal{G}\left[P_{\sigma\left(t_{0}\right)}\right]$. When $j=k_{1},\left(v_{n+i_{1}}, v_{k_{1}}\right)$ and $\left(v_{n+i_{1}}, v_{n+k_{1}}\right) \in$ $\mathcal{E}\left[P_{k_{1}}\right]$ and so $\left(v_{n+i_{0}}, v_{k_{1}}\right)$ and $\left(v_{n+i_{0}}, v_{n+k_{1}}\right)$ are edges in $\mathcal{G}\left[P_{j}\right] \circ \mathcal{G}\left[P_{\sigma\left(t_{2}\right)}\right] \circ \cdots \circ \mathcal{G}\left[P_{\sigma\left(t_{0}\right)}\right]$ since $\left(v_{n+i_{0}}, v_{n+i_{1}}\right)$ is an edge in $\mathcal{G}\left[P_{\sigma\left(t_{2}\right)}\right] \circ \cdots \circ \mathcal{G}\left[P_{\sigma\left(t_{0}\right)}\right]$. One then concludes that $\left(v_{n+i_{0}}, v_{k_{1}}\right)$ and $\left(v_{n+i_{0}}, v_{n+k_{1}}\right)$ are in $\mathcal{G}\left[P_{\sigma(t)}\right] \circ \mathcal{G}\left[P_{\sigma(t-1)}\right] \circ$ $\cdots \circ \mathcal{G}\left[P_{\sigma\left(t_{0}\right)}\right]$ for all $t \geq t_{2}$. The above argument applies to the outgoing neighbors of $i_{s}, s=1, \ldots, r$.

From the assumption that the graph $\mathcal{G}[W]$ is rooted, one knows that the vertex $s_{i_{0}}$ can reach every other vertex in $\mathcal{G}[W]$ on a path of at most $n-1$ edges. By repeatedly applying the above arguments, one can derive that the graph composition $\mathcal{G}\left[P_{\sigma\left(t_{0}+(n-1) T-1\right)}\right] \circ \cdots \circ \mathcal{G}\left[P_{\sigma\left(t_{0}\right)}\right]$ contains the directed edges $\left(v_{i_{0}}, v_{i_{0}}\right)$ and $\left(v_{i_{0}}, v_{n+i_{0}}\right)$ and $\left(v_{n+i_{0}}, v_{j}\right)$ for all $j \in\{1, \ldots, 2 n\} \backslash\left\{i_{0}\right\}$. Since $t_{0} \leq t+T-1$, one has $t_{0}+(n-1) T-1 \leq t+n T-1$. It is easy to see that for any $m_{1}, m_{2} \in \mathcal{I}$, the graph composition $\mathcal{G}\left[P_{m_{1}}\right] \circ \mathcal{G}\left[P_{\sigma\left(t_{0}+(n-1) T-1\right)}\right] \circ \cdots \circ \mathcal{G}\left[P_{t_{0}}\right] \circ \mathcal{G}\left[P_{m_{2}}\right]$ contains the directed edges $\left\{\left(v_{i_{0}}, v_{i_{0}}\right),\left(v_{i_{0}}, v_{n+i_{0}}\right),\left(v_{n+i_{0}}, v_{j}\right), j \in\right.$ $\left.\{1, \ldots, 2 n\} \backslash\left\{i_{0}\right\}\right\}$ and so does $\mathcal{G}\left[P_{\sigma(t+n T-1)}\right] \circ \cdots \circ \mathcal{G}\left[P_{\sigma(t)}\right]$.

Since $t \geq 0$ is an arbitrary time instant, using the definition of graph composition again, one has that $\mathcal{G}\left[P_{\sigma(t+2 n T-1)}\right] \circ$ $\ldots \circ \mathcal{G}\left[P_{\sigma(t)}\right]$ contains the edges $\left(v_{i_{0}}, v_{j}\right)$ for all $j \in$ $\{1, \ldots, 2 n\}$. By Lemma 3 in [27], $\mathcal{G}\left[P_{\sigma(t+2 n T-1)} \cdots P_{\sigma(t)}\right]$ has the same graph structure as $\mathcal{G}\left[P_{\sigma(t+2 n T-1)}\right] \circ \cdots \circ \mathcal{G}\left[P_{\sigma(t)}\right]$ and hence the matrix $P_{\sigma(t+2 n T-1)} \cdots P_{\sigma(t)}$ contains a positive column and is a scrambling matrix as well for all $t \geq 0$. The nonzero entry of $P_{\sigma(t+2 n T-1)} \cdots P_{\sigma(t)}$ is lower bounded by $\alpha^{2 n T}$ and its coefficient of ergodicity satisfies

$$
\tau\left(\Pi_{k=t}^{t+2 n T-1} P_{\sigma(k)}\right) \leq 1-\alpha^{2 n T} .
$$

Similarly to the proof in Theorem 1, one has that

$$
\tau\left(\Pi_{k=0}^{t} P_{\sigma(k)}\right) \leq\left(1-\alpha^{2 n T}\right)^{\frac{t}{2 n T}-1},
$$

which in turn implies

$$
\lim _{t \rightarrow \infty} \tau\left(\Pi_{k=0}^{t} P_{\sigma(k)}\right) \leq \lim _{t \rightarrow \infty}\left(1-\alpha^{2 n T}\right)^{\frac{t}{2 n T}-1}=0 .
$$

It follows that $\Pi_{k=0}^{t} P_{\sigma(k)}$ converges exponentially fast to a stochastic matrix $\mathbf{1}_{2 n} v^{\top}$, for some nonnegative $v \in$ $\mathbb{R}_{\geq 0}^{2 n}$. The equation $\zeta(t)=\prod_{k=0}^{t} P_{\sigma(k)} \zeta(0)$ then yields $\lim _{t \rightarrow \infty} \zeta(t)=c \mathbf{1}_{2 n}$, where $c=v^{\top} \zeta(0)$.

\section{Simulations}

In this section, two numerical examples are presented, first to illustrate the theoretical result, and then show more complex system dynamics. Let

$$
W=M=\left[\begin{array}{ccccc}
0.5 & 0.25 & 0 & 0.25 & 0 \\
0.4 & 0 & 0 & 0 & 0.6 \\
0 & 0.5 & 0.5 & 0 & 0 \\
0 & 0 & 0 & 0.5 & 0.5 \\
0 & 0.5 & 0 & 0 & 0.5
\end{array}\right]
$$

and thus $\mathcal{G}[W]$ is rooted at $s_{1}$. The parameters are set as $\lambda_{i}=1, \phi_{i}=0.6, \forall i=1, \ldots, 5$. We take the initial private and expressed opinions of the five individuals as $x_{i}(0)=$ $i$, and $\hat{x}_{i}(0)=i-0.1$ for all $i=1, \ldots, 5$. In the first simulation, every individual activates at least once every 12 steps, i.e., Assumption 1 holds with $T=12$. The evolution of the individuals' expressed and private opinions are shown in Fig. 1 and are observed to reach consensus and as consistent with Theorem 2.

However, Assumption 2 assumes that $\lambda_{i}=1$ for all $i \in \mathcal{I}$, i.e., no individual is attached to prejudice $u_{i}$. We conjecture that the system Eq. (5) will still converge if we relax the assumption to allow $\lambda_{i}<1$, i.e., some individuals are attached to their prejudice $u_{i}$. To illustrate this conjecture, we take $\lambda_{i}=0.3, \phi_{i}=0.6, \forall i=1, \ldots, 5$, i.e., every individual is partially attached to her prejudice. Every individual activates at least once every 12 steps, and $W$ and the initial conditions are as above. Fig. 2 shows the evolution of the five individuals' expressed and private opinions which all converge to some constants. However, different from what has been observed in Fig. 1, in this case, there is a discrepancy between each individual's expressed and private opinions and neither the expressed nor the private opinions of the individuals reach a consensus. Convergence to a persistent disagreement, and the aforementioned discrepancy between expressed and private opinions, was observed in multiple simulations, suggesting that a more general convergence result can be established that relaxes Assumption 2. The proof for the convergence of the model (2) with the parameters $\lambda_{i} \in[0,1]$ and $\phi_{i} \in[0,1]$ is a future research topic. 


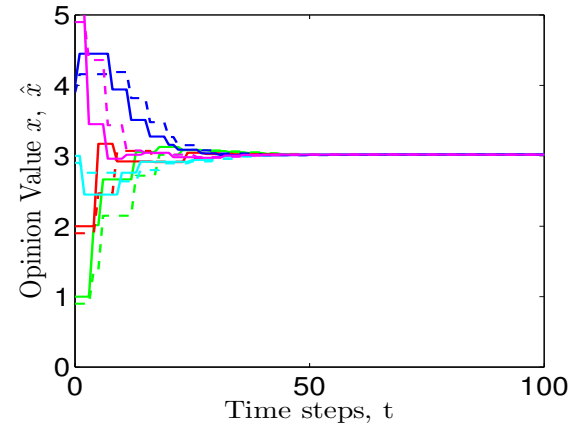

Fig. 1. The evolution of the five individuals' expressed and private opinions, where $\lambda_{i}=1, \phi_{i}=0.6$. (Solid and dashed lines represent private and expressed opinions, respectively.)

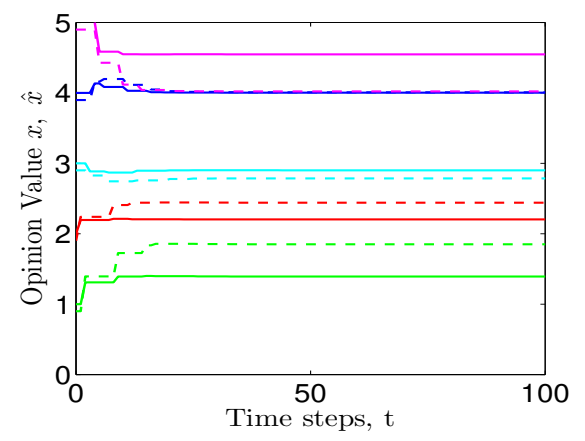

Fig. 2. The evolution of the five individuals' expressed and private opinions, where $\lambda_{i}=0.3, \phi_{i}=0.6$. (Solid and dashed lines represent private and expressed opinions, respectively.)

\section{CONCLusions}

In this paper, we have studied an asynchronous discretetime opinion dynamics model. At each time instant, a single individual updates her expressed and private opinions. By assuming all individuals activate at least once within a finite time period, and the influence network is rooted, we established convergence to consensus when no individual was attached to her prejudice. Much future work remains to be pursued. First, we aim to establish convergence under more general conditions, by allowing individuals to be attached to their prejudices. Our simulations suggest that besides convergence to a steady state of disagreement, the system can exhibit a complex phenomenon in which the steady state expressed and private opinions of the same individual are different. Moreover, we hope to establish more general convergence results in which the network influencing individuals' private opinions is different to the network determining the public opinion. That is, the private opinion of an individual may be influenced by the expressed opinion of one set of individuals, but her expressed opinion is shaped by the public opinion formed from a different set of individuals.

\section{REFERENCES}

[1] N. E. Friedkin and E. C. Johnsen, Social Influence Network Theory: A Sociological Examination of Small Group Dynamics. Cambridge University Press, 2011.
[2] M. H. DeGroot, "Reaching a consensus," Journal of the American Statistical Association, vol. 69, no. 345, pp. 118-121, 1974.

[3] N. H. Anderson, Foundations of Information Integration Theory. Academic Press New York, 1981, vol. 578.

[4] N. E. Friedkin and E. C. Johnsen, "Social influence and opinions," Journal of Mathematical Sociology, vol. 15, no. 3-4, pp. 193-206, 1990.

[5] R. Hegselmann and U. Krause, "Opinion dynamics and bounded confidence models, analysis, and simulation," Journal of Artificial Societies and Social Simulation, vol. 5, no. 3, 2002.

[6] C. Altafini, "Consensus problems on networks with antagonistic interactions," IEEE Transactions on Automatic Control, vol. 58, no. 4, pp. 935-946, 2013.

[7] W. Xia, M. Cao, and K. H. Johansson, "Structural balance and opinion separation in trust-mistrust social networks," IEEE Transcations on Control of Network Systems, vol. 3, no. 1, pp. 46-56, 2016.

[8] A. V. Proskurnikov and R. Tempo, "A tutorial on modeling and analysis of dynamic social networks. Part I," Annual Reviews in Control, vol. 43, pp. 65-79, 2017.

[9] — - "A tutorial on modeling and analysis of dynamic social networks. Part II," Annual Reviews in Control, vol. 45, pp. 166-190, 2018.

[10] S. E. Asch, Groups, Leadership, and Men, H. S. Guetzkow, Ed. Carnegie Press: Pittsburgh, 1951

[11] T. Kuran, Private Truths, Public Lies: the Social Consequences of Preference Falsification. Harvard University Press, 1997.

[12] R. B. Cialdini and N. J. Goldstein, "Social influence: Compliance and conformity," Annual Reviews in Psychology, vol. 55, pp. 591-621, 2004.

[13] P. Duggins, "A psychologically-motivated model of opinion change with applications to american politics," Journal of Artificial Societies and Social Simulation, vol. 20, no. 1, pp. 1-13, 2017.

[14] M. Ye, Y. Qin, A. Govaert, B. D. O. Anderson, and M. Cao, "An influence network model to study discrepancies in expressed and private opinions," Automatica, vol. 107, no. 7, pp. 371-381, Sep. 2019

[15] M. Ye, Opinion dynamics and the evolution of social power in social networks. Springer, Cham, 2019.

[16] C. Cheng and C. Yu, "Opinion dynamics with bounded confidence and group pressure," Physica A: Statistical Mechanics and its Applications, vol. 532, p. 121900, 2019.

[17] Y. Shang, "Resilient consensus for expressed and private opinions," IEEE Transactions on Cybernetics, 2019.

[18] C. Ravazzi, P. Frasca, R. Tempo, and H. Ishii, "Ergodic randomized algorithms and dynamics over networks," IEEE Transactions on Control of Network Systems, vol. 2, no. 1, pp. 78-87, 2015.

[19] D. Acemoglu, G. Como, F. Fagnani, and A. Ozdaglar, "Opinion fluctuations and disagreement in social networks," Mathematics of Operations Research, vol. 38, pp. 1-27, 2013.

[20] W. Xia and M. Cao, "Sarymsakov matrices and asynchronous implementation of distributed coordination algorithms," IEEE Transcations on Automatic Control, vol. 59, no. 8, pp. 2228-2233, 2014.

[21] S. E. Parsegov, A. V. Proskurnikov, R. Tempo, and N. E. Friedkin, "Novel multidimensional models of opinion dynamics in social networks," IEEE Transactions on Automatic Control, vol. 62, no. 5, pp. 2270-2285, 2017

[22] G. Deffuant, D. Neau, F. Amblard, and G. Weisbuch, "Mixing beliefs among interacting agents," Advances in Complex Systems, vol. 3, no. 01n04, pp. 87-98, 2000.

[23] E. Seneta, Non-negative Matrices and Markov Chains. New York: Springer-Verlag, 1981.

[24] R. L. Gorden, "Interaction between attitude and the definition of the situation in the expression of opinion," American Sociological Review, vol. 17 , no. 1 , pp. 50-58, 1952.

[25] D. G. Taylor, "Pluralistic ignorance and the spiral of silence: A formal analysis," Public Opinion Quarterly, vol. 46, no. 3, pp. 311-335, 1982.

[26] L. Festinger, "Informal social communication," Psychological Review, vol. 57 , no. 5 , p. $271,1950$.

[27] M. Cao, A. S. Morse, and B. D. O. Anderson, "Reaching a consensus in a dynamically changing environment: A graphical approach," SIAM Journal on Control and Optimization, vol. 47, no. 2, pp. 575-600, 2008 